\title{
Osteomyelitis caused by Sporothrix schenckii in an immunocompetent patient
}

\author{
Laura Cunha Ferreira ${ }^{[1]}$, Paulo Feijo Barroso ${ }^{[1],[2],}$ Elise Tonomura ${ }^{[3]}$, \\ Tiyomi Akiti ${ }^{[4]}$ and Káris Maria de Pinho Rodrigues ${ }^{[2]}$
}

\begin{abstract}
[1]. Serviço de Doenças Infecciosas e Parasitárias, Hospital Universitário Clementino Fraga Filho, Universidade Federal do Rio de Janeiro, Rio de Janeiro, Rio de Janeiro, Brasil. [2]. Departamento de Medicina Preventiva, Faculdade de Medicina, Universidade Federal do Rio de Janeiro, Rio de Janeiro, Rio de Janeiro, Brasil. [3]. Serviço de Radiologia, Hospital Universitário Clementino Fraga Filho, Universidade Federal do Rio de Janeiro, Rio de Janeiro, Rio de Janeiro, Brasil. [4]. Serviço de Micologia, Hospital Universitário Clementino Fraga Filho,

Universidade Federal do Rio de Janeiro, Rio de Janeiro, Rio de Janeiro, Brasil.
\end{abstract}

\begin{abstract}
Sporotrichosis is the most common subcutaneous mycosis in South America and its association with zoonotic transmission remains a relevant public health problem in Rio de Janeiro, Brazil. The disease most commonly presents as subacute or chronic cutaneous lesions, although dissemination to various organs and systems occurs in rare cases, mainly in immunosuppressed individuals. This report describes a case of sporotrichosis with severe bone and subcutaneous damage in an immunocompetent patient who did not exhibit the characteristic skin lesions of sporotrichosis, including ulcers, nodules, and lymphangitis.
\end{abstract}

Keywords: Sporotrichosis. Osteomyelitis. Cutaneous fistulae.

\section{INTRODUCTION}

Sporotrichosis is a subacute or chronic infection caused by one of the six putative phylogenetic species of the Sporothrix schenckii complex ${ }^{(1)}$, a thermally dimorphic fungus that is commonly found on decaying plants and in the soil ${ }^{(2)}$. The disease occurs worldwide, although it is more common in tropical and subtropical areas with warm $\left(15-25^{\circ} \mathrm{C}\right)$ and humid $(90 \%)$ climates. Sporotrichosis is the most common subcutaneous mycosis in South America and has been reported in Brazil since $1907^{(3)}$, where epidemics have been related to close contact with infected cats ${ }^{(4)}$. The disease is initiated when the fungus enters a minor skin injury or, rarely, when the spores of the fungus are inhaled ${ }^{(5)}$. A rare case of osteoarticular sporotrichosis in an immunocompetent patient is presented herein.

\section{CASE REPORT}

A 35-year-old black man, who was born and residing in the neighborhood of Riachuelo in Rio de Janeiro (Brazil), presented for care in July 2014.

At the first clinical appointment, the patient reported that a year previously he had presented with a painful edema in the dorsum of the right foot associated with progressive swelling,

Corresponding author: Profa. Káris Maria de Pinho Rodrigues. e-mail: karis@cives.ufrj.br

Received 13 December 2015

Accepted 14 March 2016 which had evolved to spontaneous drainage of secretions a few weeks later. The patient denied any previous skin lesions. Over the following months, the pretibial region $(6-8 \mathrm{~cm}$ in length) and right ankle had become edematous and showed spontaneous discharge (Figures 1A and 1B). Notably, the patient did not exhibit the classic skin lesions of sporotrichosis, including ulcers, nodules, and lymphangitis. The patient reported a $10 \mathrm{~kg}$ weight loss over the preceding year, and a daily fever for the prior 6 months.

A healthy cat had lived in the patient's house until its death 3 years previously, although this cat had never presented with any skin injuries.

Magnetic resonance imaging (SIEMENS Avanto 1,5T MR system) showed osteomyelitis with severe damage to the right tibia (Figures 2A and 2B) and talus (Figure 2C). Bone fistulas in the adjacent tissue were also detected (Figure 2A and 2B).

Percutaneous aspirations of the lesions in the right pretibial area and foot were performed for diagnostic evaluation. Parker ink, Gram, and Ziehl-Neelsen staining were all negative for microorganisms. Cultures under anaerobic conditions and on media for Mycobacterium revealed no bacterial growth. Samples cultured on Sabouraud Dextrose Agar 2\% (DIFCO) containing chloramphenicol, Mycosel Agar (BD-BBL) and Yeast extract Agar media (DIFCO) for 5 days at $25^{\circ} \mathrm{C}$ all revealed growth of lobulated, cream-colored, smooth colonies with the characteristic hyphal form of a fungus belonging to the $S$. schenckii complex. The characteristic dimorphism, with appearance of yeast forms, was observed following incubation on Sabouraud Dextrose Agar at $37^{\circ} \mathrm{C}$. 


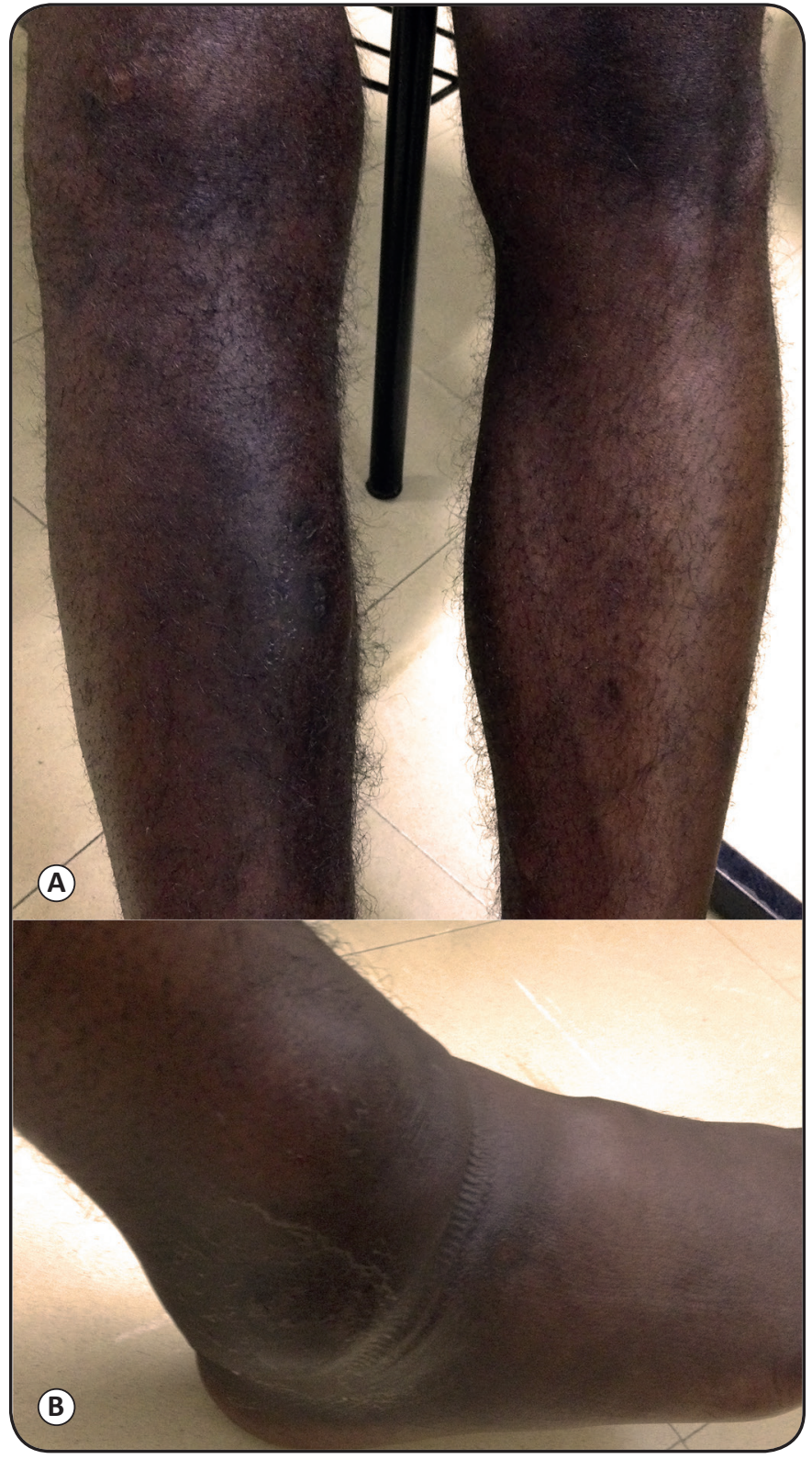

FIGURE 1. Images of the (A) right leg (B) and ankle before treatment. Edema was observed in the dorsum of the right foot, ankle, and pretibial region from where the purulent material was obtained for culture.

Treatment with $400 \mathrm{mg}$ once daily itraconazole was initiated in July 2014, and, after 7 months of therapy, the fistulas and edema were totally regressed. However, the bone damage remained (Figure 3A, 3B and 3C), and the patient continued treatment with Itraconazole.

\section{Ethical considerations}

The patient was informed and agreed with the report.

\section{DISCUSSION}

The most frequent presentation of sporotrichosis is either a subacute or a chronic, ulcerated and/or verrucous, erythematous nodule at the site of entry of the fungus into the

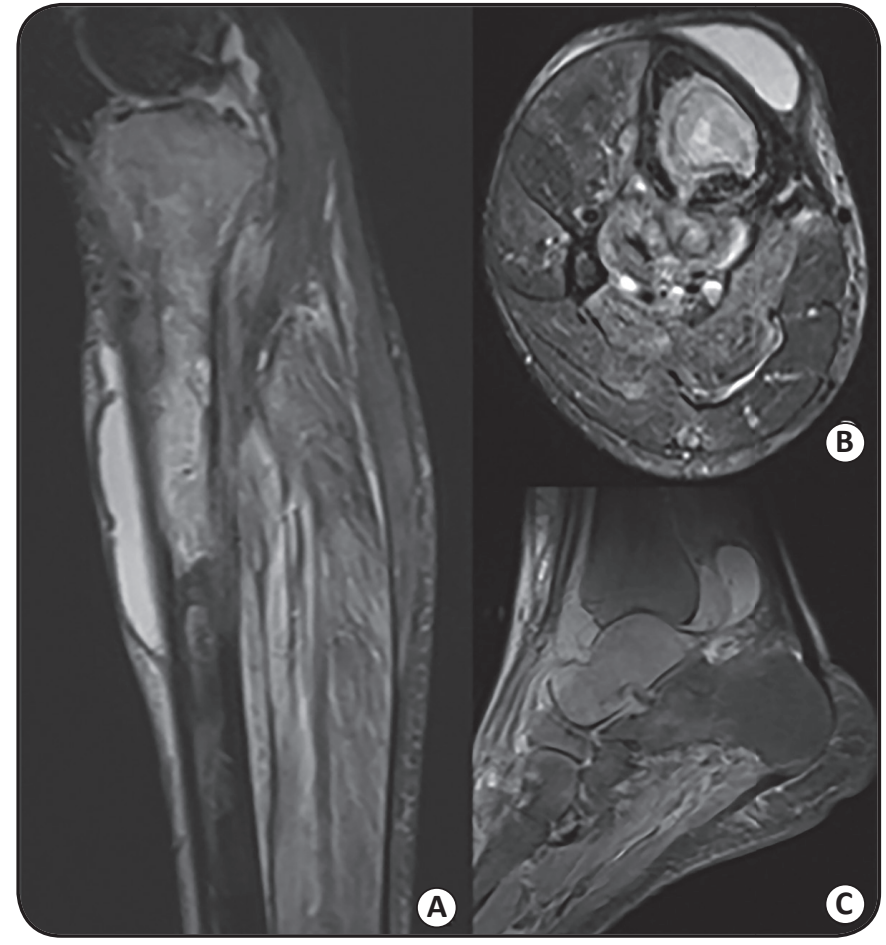

FIGURE 2. Magnetic resonance imaging of the right lower extremity before treatment. (A) Sagittal and (B) Axial short tau inversion recovery images of the tibia showed areas of abnormally high signal intensity in the bone marrow, fistula drainage into the posterior adjacent tissue, and abscess formation in the pretibial space. (C) A sagittal proton density image of the ankle showed the abnormal high signal intensity of the talus bone marrow and fluid with varying signals within the articular space and the adjacent soft tissues, indicating a distinct protein content.

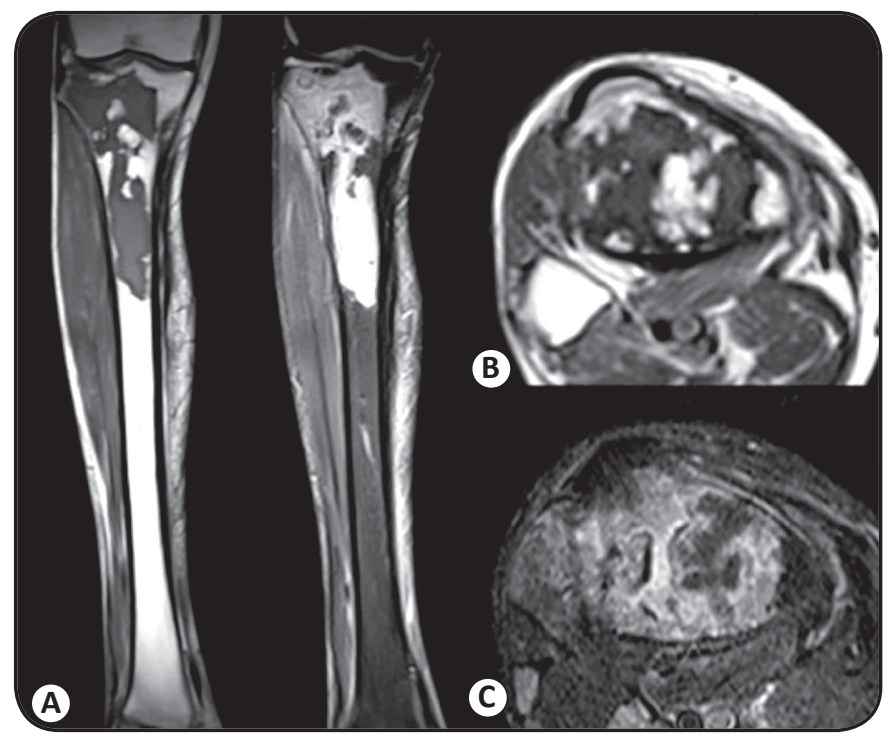

FIGURE 3. Magnetic resonance imaging of the right lower extremity at 7 months following initiation of Itraconazole therapy. (A) Sagittal and (B) Axial short tau inversion recovery images of the tibia showed areas of abnormally high signal intensity in the bone marrow, with clearing of the soft tissue abscess. (C) A sagittal proton density image of the ankle showed the abnormal high signal intensity of the talus bone marrow and fluid with varying signals within the articular space and the adjacent soft tissues, indicating a distinct protein content. 
skin, with or without local lymphatic spread ${ }^{(4)}$. The dissemination to viscera or osteoarticular structures, although uncommon, has been described and is associated with alcohol abuse or immunosuppression, especially in human immunodeficiency virus (HIV)-infected patients with advanced immunosuppression ${ }^{(6)}$. Bone and articular involvement are the most common presentations of extracutaneous sporotrichosis ${ }^{(7)}$, and can occur via direct inoculation of the fungus or through hematogenous dissemination. The most commonly affected bones are the tibia, small bones of the hands, radius, ulna, knee, and ankle ${ }^{(8)}$.

Notably, this patient showed aggressive clinical progression, with extensive osteoarticular damage, fistulae, and systemic symptoms, although without the initial cutaneous lesions that characterize sporotrichosis. In addition, the patient was not HIV-positive and did not have any other known reason for immunosuppression. The patient reported no contact with an infected animal.

This article highlights the importance of considering sporotrichosis as a diagnostic possibility in chronic osteomyelitis, even in the absence of skin lesions or a suggestive epidemiological history. It has been suggested that a failure to consider S. schenckii as a potential etiologic agent in these settings may lead to an average delay in diagnosis of 25 months, with eventual progression to dissemination and death ${ }^{(9)}$. The diagnostic delay in this case is likely responsible for the duration and severity of the damage observed in the bones; however, we cannot eliminate the possibility that the disease was caused by Sporothrix brasiliensis, which is considered the most virulent of the S. schenckii complex, and has been isolated in Rio de Janeiro previously ${ }^{(10)}$. Other agents, including Mycobacterium species, Actinomyces, Nocardia, and the fungi responsible for the eumycotic mycetomas, are known to cause cutaneous and osteoarticular infections with similar clinical manifestations to those described in this patient, and must be included in the differential diagnosis ${ }^{(11)}$. It is important to be aware of these possibilities, and to look for the characteristic clear sulfur granules present in the purulent secretions of actinomycosis, and less frequently in nocardiosis, as well as the black or pale grains present in eumycotic mycetomas. Granules can be examined by microscopy to reveal the morphology of the bacteria or fungi present in the lesion, or by culture, which is more accurate in revealing the causative agent, but can take as long as 4 weeks to reveal growth in the case of some fungi ${ }^{(12)(13)}$. The material should also be cultivated in anaerobic media for isolation of Actinomyces and Nocardia ${ }^{(12)}$. To confirm a sporotrichosis diagnosis, serological analyses and direct stains are generally not useful. Culture remains the gold standard and most sensitive method, although one negative culture does not always eliminate the diagnosis. In specific settings, particularly when there is joint drainage or when synovial biopsies are not available, repeated samples may be required to prevent diagnosis delay ${ }^{(11)}$.

Cutaneous and lymphocutaneous sporotrichosis can be treated with a saturated solution of potassium iodide or itraconazole, which is preferable due to its better tolerability and efficacy. Furthermore, when the infection affects osteoarticular structures, itraconazole is the therapy of choice. However, amphotericin B should be used when the disease is disseminated and associated with immunosuppression ${ }^{(14)}$. Itraconazole was used in this patient and resulted in total resolution of the fistulae and edema. However, the maintenance of bone damage demanded longer-term treatment and demonstrated the severe consequences of a delayed diagnosis.

\section{Acknowledgments}

We would like to thank Maria da Gloria Carvalho Barreiros, whose work was invaluable for the isolation of the fungus.

\section{Conflicts of Interest}

The authors declare that there is no conflict of interest.

\section{REFERENCES}

1. Marimon R, Gené J, Cano J, Trilles L, Lazera MS, Guarro J. Molecular phylogeny of Sporothrix schenckii. J Clin Microbiol 2006; 44:3251-3256.

2. Zhang YQ, Xu XG, Zhang M, Jiang P, Zhou XY, Li ZZ, et al. Sporotrichosis: clinical and histopathological manifestations. Am J Dermatopathol 2011; 33:296-302.

3. Ramos-e-Silva M, Vasconcelos C, Carneiro S, Cestari T. Sporotrichosis. Clin Dermatol 2007; 25:181-187.

4. Barros MBL, Schubach AO, Valle ACF, Galhardo MCG, Conceição-Silva F, Schubach TMP, et al. Cat-transmitted Sporotrichosis epidemic in Rio de Janeiro, Brazil: description of a series of cases. Clin Infect Dis 2004; 38:529-535.

5. Conti Díaz IA. Epidemiology of sporotrichosis in Latin America. Mycopathologia 1989; 108:113-116.

6. Mercurio MG, Elewiski BE. Therapy of sporotrichosis. Semin Dermatol 1993; 12:285-289.

7. Wilson DE, Mann JJ, Bennett JE, Utz JP. Clinical features of extracutaneous sporotrichosis. Medicine (Baltimore) 1967; 46:265-279.

8. Appenzeller S, Amaral TN, Amstalden EMI, Bertolo MB, Marques Neto JF, Samara AM, et al. Sporothrix schenckii infection presented as monoarthritis: report of two cases and review of the literature. Clin Rheumatol 2006; 25:926-928.

9. Rex JH, Okhuysen PC. Sporothrix schenckii. In: Bennett JE, Dolin R, Blaser MJ, editors. Mandell, Douglas, and Bennett's Principles and Practice of Infectious Diseases, $8^{\text {th }}$ edition, Philadelphia, PA, Elsevier/Saunders 2014. p. 2920-2924.

10. Almeida-Paes R, Oliveira MME, Freitas DFS, Valle ACF, ZacopéOliveira RM, Gutierrez-Galhardo MC. Sporotrichosis in Rio de Janeiro, Brazil: Sporothrix brasiliensis is associated with atypical clinical presentations. PLoS Negl Trop Dis 2014; 8:e3094

11. Kwon-Chung KJ, Bennett JE. Sporotrichosis. In: Rippon JW, editor. Medical Mycology. Philadelphia: Lea e Fibeger, 1992. p. 707-729.

12. Yousif BM, Fahal AH, Shakir MY. A new technique for the diagnosis of mycetoma using fixed blocks of aspirated material. Trans R Soc Trop Med Hyg 2010;104:6-9.

13. Russo TA. Agents of Actinomycosis. In: Bennett JE, Dolin R, Blaser MJ, editors. Mandell, Douglas, and Bennett's Principles and Practice of Infectious Diseases, $8^{\text {th }}$ edition, Philadelphia, PA, Elsevier/Saunders 2014 p 2864-2873.

14. Kauffman CA, Bustamante B, Chapman SW, Pappas PG, Infectious Diseases Society of America. Clinical practice guidelines for the management of sporotrichosis: 2007 update by the Infectious Diseases Society of America. Clin Infect Dis 2007;45:1255-1265. 